per cent. of those children who have defective hearing have often been found to be dullards. Also those suffering from adenoid growths are likely to be found in the class of dull children. And while myopic children are often found among thase more precocious and studious in school work, this due, perhaps, to their lack of normal interest in things out of doors and muscular activities, those with eye defects often seem hopelessly dull.

It is evident that we are dealing with a problem fundamental in pedagogy and school hygiene. Every parent knows the leaden stupidity that at times comes over children, and every teacher has doubtless had experiences with at least a few cases of it in chronic form. This is the one defect which to many teachers seems hopeless. The only redeeming thing about stupidity seems to have been discovered by a German, who with rather a labored attempt at wit has said that the stupid children will make invincible soldiers, because the gods themselves fight in vain against stupidity; but what is impossible to the gods of pedagogy is sometimes possible to Hygeia. When stupidity is due to a defect of the sense organs, the difficulty can sometimes be removed by the simple device of seating the pupil in a favorable position; a surgical operation for an adenoid growth has removed the cause of stupidity in the case of many children; and frequently what the stupid child specially needs is enough to eat, or sufficient sleep, or rest from work imposed out of school hours, or perhaps the mere stimulus of social success. In any case the cause should be sought.

Thus the simple problem with which we started leads out into the wider problems of social hygiene and social pedagogy ; and here I must leave it with the hope that it will be considered by teachers and studied further by investigators.

WM. H. BuRNHAM

Crabi University

\section{THE PRINCIPLE OF RELATIVITY}

$A T$ the recent Boston meeting of the American Physical Society there was so much general interest in the principle of relativity and so many questions were asked me personally by those who had given the subject very little attention, that it seems timely to give a brief introduction to the subject on a somewhat simpler basis than I think has yet been attempted. The method employs several of the "non-mathematical" conceptions first introduced by Lewis and Tolman, but I think the demonstrations will be found even simpler than theirs.

The principle of relativity is one attempt, and by far the most successful attempt as yet, to explain the failure of all experiments designed to detect the earth's motion through space, by its effect on terrestrial phenomena. It generalizes this universal negative result into its first postulate, which is, the uniform translatory motion of any system can not be detected by an observer traveling with the system and making observations on it alone.

The second postulate is that the velocity of light is independent of the relative velocity of the source of light and observer.

At the very outset, it is important to realize that we have no long-standing experience with systems moving with velocities comparable with that of light, and therefore that primitive intuition may not be the very best guide in first introducing us to them. We might easily imagine a peasant scorning the suggestion that the dimensions of a rigid body changed with the temperature, and declaring, on being 
pressed that such an idea was clearly against common sense.

The whole principle of relativity may be based on an answer to the question: When are two events which happen at some distance from each other to be considered simultaneous? The answer, "When they happen at the same time," only shifts the problem. The question is, how can we make two events happen at the same time when there is a considerable distance between them.

Most people will, I think, agree that one of the very best practical and simple ways would be to send a signal to each point from a point half-way between them. The velocity with which signals travel through space is of course the characteristic "space velocity," the velocity of light.

Two clocks, one at $A$ and the other at $B$, can therefore be set running in unison by means of a light signal sent to each from a place midway between them.

Now suppose both clock $A$ and clock $B$ are on a kind of sidewalk or platform moving uniformly past us with velocity $v$. In Fig. 1 (2) is the moving platform and (1) is the fixed one, on which we consider ourselves placed. Since the observer on platform (2) is moving uniformly he can have no reason to consider himself moving at all, and he will use just the method we have indicated to set his two clocks $A$ and $B$ in unison. He will, that is,

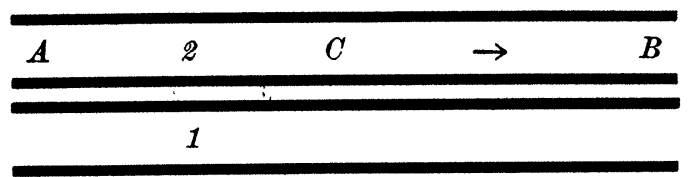

FIG. 1

send a light flash from $C$, the point midway between $A$ and $B$, and when this flash reaches the two clocks he will start them with the same reading.

To us on the fixed platform, however, it will of course be evident that the clock $B$ is really a little behind clock $A$, for, since the whole system is moving in the direction of the arrow, light will take longer to go from $C$ to $B$ than from $C$ to $A$. Thus the clock on the moving platform which leads the other will be behind in time.

Now it is very important to see that the two clocks are in unison for the observer moving with them (in the only sense in which the word "unison" has any meaning for him), for if we adopt the first postulate of relativity, there is no way in which he can know that he is moving. In other words, he has just as much fundamental right to consider himself stationary as we have to consider ourselves stationary, and therefore just as much right to apply the midway signal method to set his clocks in unison as we have in the setting of our "stationary clocks." "Stationary" is, therefore, a relative term and anything which we can say about the moving system dependent on its motion, can with absolutely equal right be said by the moving observer about our system.

We are, therefore, forced to the conclusion that, unless we discard one of the two relativity postulates, the simultaneity of two distant events means a different thing to two different observers if they are moving with respect to each other.

The fact that the moving observer disagrees with us as to the reading of his two clocks as well as to the reading of two similar clocks on our "stationary" platform, gives us a complete basis for all other differences due to point of view.

A very simple calculation will show that the difference in time between the two moving clocks is ${ }^{1}$

${ }^{1}$ The time it takes light to go from $C$ to $B$ is $\frac{1}{2} /(V-v)$ and the time to go from $C$ to $A$ is $\frac{1}{2} /(V+v)$. The difference in these two times is the amount by which the clocks disagree and this 
where

$$
1 / V \beta /\left(1-\beta^{2}\right)
$$

$$
\begin{aligned}
& l=\text { distance between clocks } A \text { and } B ; \\
& v=\text { velocity of moving system; } \\
& V=\text { velocity of light; } \\
& \beta=v / V .
\end{aligned}
$$

The way in which this difference of opinion with regard to time between the moving ing observer and ourselves leads to a difference of opinion with regard to length also may very easily be indicated as follows:

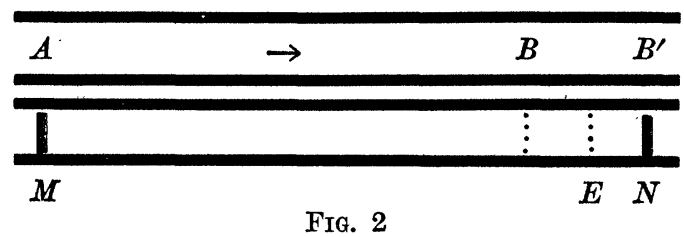

Suppose the moving observer desires to let us know the distance between his clocks and says he will have an assistant stationed at each clock and each of these, at a given instant, is to make a black line on our platform. He will, therefore, he says, be able to leave marked on our platform an exact measure of the length between his clocks and we can then compare it at leisure with any standard we choose to apply.

We, however, object to this measure left with us, on the ground that the two assistants did not make their marks simultaneously and hence the marks left on our platform do not, we say, represent truly the distance between his clocks. The difference is readily shown in Fig. 2, where $M$ represents the black mark made on our platform at a certain time by the assistant at $A$, and $N$ that made by the assistant at $B$ at a later time. The latter assistant waited, we say, until his clock read the same as clock $A$, waited, that is, until $B$ was at $B^{\prime}$; and then made the mark $N$. The moving observer declares, therefore, that the distance $M N$ is equal to the disdifference becomes, on simplification, the expression given above. tance $A B$, while we say that $M N$ is greater than $A B$.

Again it must be emphasized that, because of the first fundamental postulate, there is no universal standard to be applied in settling such a difference of opinion. Neither the standpoint of the "moving" observer nor our standpoint is wrong. The two merely represent two different sides of reality. Any one could ask: What is the "true" length of a metal rod? Two observers working at different temperatures come to different conclusions as to the "true length." Both are right. It depends on what is meant by "true." Again, asking a question which might have been asked centuries ago, is a man walking toward the stern of an eastbound ship really moving west? We must answer "that depends" and we must have knowledge of the questioner's view-point before we can answer yes or no.

A similar distinction emerges from the principle of relativity. What is the distance between the two clocks? Answer: that depends. Are we to consider ourselves with the clock system when we answer, or passing the clocks with a hundredth the velocity of light or passing the clocks with a tenth the velocity of light? The answer in each case must be different, but in each case may be true.

It must be remembered that the results of the principle of relativity are as true and no truer than its postulates. If future experience bears out these postulates then the length of the body, even of a geometrical line, in fact the very meaning of "length," depends on the point of view, that is, on the relative motion of the observer and the object measured. The reason this conclusion seems at first contrary to common sense is doubtless because we, as a race, have never had occasion to observe directly velocities high enough to 
make such effects sensible. The velocities which occur in some of the newly investigated domains of physics are just as new and outside our former experience as the fifth dimension.

Returning now to the magnitude of this difference of opinion as to the distance between the clocks, it is easy to show that, from our point of view, the moving observer overestimates the distance in the ratio

$$
1 /\left(1-\beta^{2}\right) \text {. }
$$

So that it may be said in general that lengths in the direction of motion, which he says are equal, we say are unequal in this same ratio.

On lengths perpendicular to the direction of motion our estimates agree.

Now let us ask ourselves: What are corresponding lengths in the two systems? Corresponding lengths may with propriety be given the same name, "meter" for instance. The condition that two lengths should be "corresponding" is simply that each observer comes to the same conclusion with respect to the other length.

The lengths $A B$ and $M N$ are not "corresponding," for the moving observer says that $M N$ is equal to $A B$, while we say $A B$ is less than $M N$, in the ratio $\left(1-\beta^{2}\right)$. If, however, we mark off on our platform a length which is a mean proportion between our estimate of the length $A B$ and the length $M N$, this length, say $M E$, will "correspond" to the length $A B$, for we shall then say, that $A B$ is less than $M E$ in the ratio $\sqrt{1-\beta^{2}}$, while the moving observer will say that $M E$ is less than $A B$ in the same ratio.

Thus any length, in the direction of motion, on a moving system is estimated less in the ratio $\sqrt{1-\beta^{2}}$ by a "stationary" observer.

Or, put in a better way, an observer viewing a system which is moving with re- spect to him, sees all lengths, in the direction of motion, shrunken in the proportion $\sqrt{1-\beta^{2}}$, where $\beta$ is velocity with which the system is passing him in terms of the velocity of light.

We have now reached two results, which we may summarize thus; first, clocks which a moving observer calls in unison do not appear in unison to a "stationary" observer, the clock in advance as regards motion appearing behind the other in time, and second, distances in the moving system appear shortened in the direction of motion in the ratio $\sqrt{1-\beta^{2}}$. In the above we can, of course, interchange the words "moving", and "stationary."

Next let us turn our attention to the unit of time in each system. It is not hard to show that the unit of time in the moving system will appear to us greater than ours in the ratio $1 / \sqrt{1-\beta^{2}}$. This is due to the fact that in the moving system forward clocks are behind in time.

In the measurement of time we assume a certain standard motion to be taking place at a constant rate and then take as a measure of time the total displacement which this motion has caused. Time measurement with an ordinary clock is obviously a special case of this general rule.

The moving observer can adopt as his unit of time the time it takes light, moving with the characteristic ${ }^{2}$ space velocity $V$, to travel a certain distance $d$ and return to him.

Suppose $d$ is in the direction of motion, and the light after traveling a certain distance in the direction of motion is reflected back to the observer. He will then write

$$
t=d / V \text {. }
$$

We, however, "know" that he is overestimating the distance $d$ in the ratio $1 / \sqrt{1-\beta^{2}}$

${ }^{2}$ That the moving observer's estimate of $V$ can not change with his velocity follows of course from the first postulate. 
and overestimating also the average velocity with which his signal travels through his system in the ratio $1 /\left(1-\beta^{2}\right),{ }^{3}$ thus he is underestimating his time in the ratio $\sqrt{1-\beta^{2}}$. A certain time interval, that is, appears less to him than to us and hence his unit of time appears to us greater than ours in the ratio $1 / \sqrt{1-\beta^{2}}$.

This paper has become long enough without an attempt to discuss the units of mass and force. It has been my purpose merely to answer a number of questions which the experience of the Boston meeting led me to believe were in the minds of many who had not given the subject enough thought to understand easily the more profound discussions.

The apparent transverse mass is, I think, best derived by Lewis and Tolman ${ }^{4}$ in their excellent paper on the principle of relativity, and the relation between transverse and longitudinal mass is shown in the most direct and simple way by Bumstead ${ }^{5}$ making use of the torsion pendulum. Any one interested in the subject should read these two papers.

It is, of course, true that the principle of relativity has a much deeper logical signifcance than the simple, more or less concrete conceptions on which it is based in the present paper would lead one to suppose, but in an introduction to such a subject concreteness may not be a fault.

It should be restated that the results of the principle for uniform translation are

\footnotetext{
${ }^{3}$ The average velocity of a signal traveling through his system with a velocity which we estimate as $\nabla-v$ in one direction and $\nabla+v$ in the other, is of course obtained by dividing the total distance by the total time. The total time is obviously

$t=\frac{1}{2}$ distance $/(\nabla-v)+\frac{1}{2}$ distance $/(\nabla+v)$, and hence the average velocity is

$$
\nabla_{a}=\nabla\left(1-\beta^{2}\right) \text {. }
$$

4 Phil. Mag., 18, 510-523, 1909.

'Am. Jour. of Science, 26, pp. 493-508, 1909.
}

simply as true as its two postulates. If either of these postulates be proved false in the future, then the structure erected can not be true in its present form. The question is, therefore, an experimental one.

I think it may be said with fairness, however, that the principle is already in harmony with so many phenomena that the burden of proof lies with those who object to it. Besides the negative result of experiments to detect the earth's motion the principle is supported directly by the recent experiment of Bucherer, ${ }^{6}$ and by the still more recent experiment of Hupka. ${ }^{7}$ Indirect support is also given by Lewis' $\mathrm{s}^{8}$ independently derived theory of non-Newtonian mechanics, which agrees exactly with relativity results, and by Comstock's ${ }^{9}$ deductions from orthodox electromagnet theory which lead to conclusions so nearly coincident with those of relativity as to be very suggestive.

In closing, a word should be said with regard to the "addition of velocities" according to relativity rules. It will be evident on a little thought that if the moving platform of Fig. 1, which is passing us with velocity $v$, has on it a body traveling over it in the direction of its motion with velocity $v$ (that is, with a velocity which the observer on the moving platform calls $v$ ), then our estimate of the velocity of the body will not be $v+v_{1}$. The reason is of course that $v+v_{1}$ is the sum of two quantities, one of which is estimated by us and the other by the moving observer. We should, therefore, be inconsistent because we should have mixed view-points. Our estimate of the platform's velocity plus our estimate of the body's velocity with respect to the platform equals our estimate of the

A Ann. d. Phys., 28, S. 513-536, 1909.

'Ann. d. Phys., 31, S. 169-204, 1910.

${ }^{8}$ Phil. Mag., 16, pp. 705-717, 1908.

'Phil. Mag., 15, pp. 1-20, 1908. 
body's velocity. In this last case we have stuck to one point of view and obtained a correct result.

This feature connected with the so-called "addition of velocities" is what Professor Michelson and others so strongly object to in the relativity principle, but the result is a perfectly natural one as soon as we have seen the admissibility of more than one point of view and the difference in estimates caused thereby.

\section{F. Cомsтоск}

Massachusetts Institute

of TEChNOLOGY

SOME CONSIDERATIONS AS TO THE NATURE

OF COMETS AND THEIR PROBABLE RELATION TO THE SUN

THE ideas herein put forward are not all original with the author, though it is believed some of them may be. It is hoped that the considerations may, however, help to a simple rational understanding of the major facts regarding the behavior of comets.

The exceedingly high temperature of the sun causes it to be surrounded by an atmosphere of vapors. Some of the vaporized matter condenses in the outermost layers and eruptions are constantly occurring which partly fill the space around it with very fine particles, the smaller of which are repelled by the pressure of the sun's radiation, which pressure even overcomes the gravitative force of the sun itself. These ejected particles probably constitute the streamers which are visible during total eclipses as extending from the sun to immense distances. What we see is the effect of innumerable overlapping streams. Their extreme tenuity is evidenced by the comparatively feeble luminosity in spite of the great depth of the flux which we are at any time observing. This depth is, of course, greater than the diameter of the sun. Such coronal streamers are by no means uniformly distributed about the sun, but in certain directions, varying continually, may be more dense than in others, coinciding perhaps with great eruptive areas of the sun's surface.
It probably happens that when the outbreak is unusually violent, and when the earth happens to be passing through that part of space occupied by an abnormally extended streamer, an aurora of greater or less intensity or duration may attend the sweeping of the earth by such a streamer. The particles are probably ions or carry electric charges, and induced auroral streamers in the earth's atmosphere are for the time being visible on its dark side away from the sun.

It has been thought that comets may act in a somewhat similar way to disclose the condition of the ejected material of the sun, or, as may be conceived, to disclose a stratification or unevenness of distribution of the ejected matter from the sun. Since there is reason to believe that much of this matter is in a highly electrified state, it is not to be doubted that electrical phenomena are at the same time produced, with accompanying evolution of light. Indeed, in the free space around the sun, there must be a great intensity of ultra-violet radiation which of itself would cause emission of negative ions from matter in its path and produce electrical disturbances. But aside from this possibility, the comet is recognized as an assemblage of particles larger or smaller, moving in an orbit which involves great variations of its distance from the sun. In passing through the depths of space far away from the sun, these parts or particles may tend, by their very feeble gravitative effect, to gather up any finer particles which, on account of the intense cold of space, are substantially solid, even though at ordinary temperatures they would be gaseous. The parts of the comet's nucleus more or less porous would in this way accumulate upon their surfaces and in their pores occluded gases, condensed material and fine dust, and there would be a period of many years in which this gathering-up process, as in the case of Donati's and other long-period comets, could occur. Let a comet as an assemblage of such small masses after its long course through remote space, during which it has gathered fine particles ejected from the sun or from other bodies, reach, in 